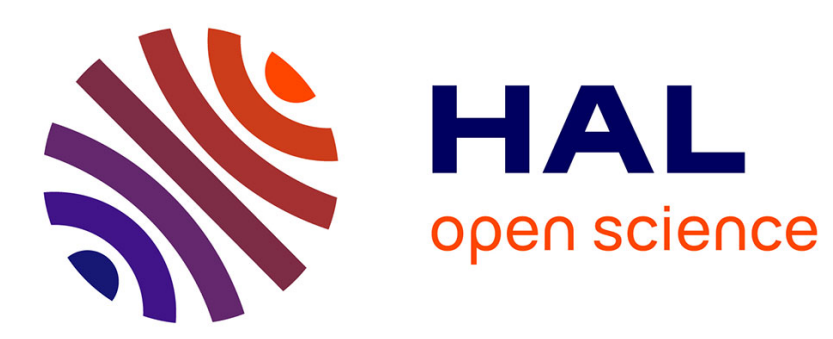

\title{
Etudes d'interactions acoustiques non-linéaires dans les jets confinés, par la fonction de bicohérence
}

\author{
R. Henry, C. Favé, S. Soreefan, A. Robins
}

\section{To cite this version:}

R. Henry, C. Favé, S. Soreefan, A. Robins. Etudes d'interactions acoustiques non-linéaires dans les jets confinés, par la fonction de bicohérence. Journal de Physique IV Proceedings, 1994, 04 (C5), pp.C5-959-C5-962. 10.1051/jp4:19945210 . jpa-00252895

\section{HAL Id: jpa-00252895 \\ https://hal.science/jpa-00252895}

Submitted on 1 Jan 1994

HAL is a multi-disciplinary open access archive for the deposit and dissemination of scientific research documents, whether they are published or not. The documents may come from teaching and research institutions in France or abroad, or from public or private research centers.
L'archive ouverte pluridisciplinaire HAL, est destinée au dépôt et à la diffusion de documents scientifiques de niveau recherche, publiés ou non, émanant des établissements d'enseignement et de recherche français ou étrangers, des laboratoires publics ou privés. 


\title{
Etudes d'interactions acoustiques non-linéaires dans les jets confinés, par la fonction de bicohérence
}

\author{
R. HENRY, C. FAVÉ, S. SOREEFAN et A. ROBINS
}

Laboratoire d'Etudes Aérodynamiques, URA 191, Bât. H, Université de Poitiers, 40 avenue du Recteur Pineau, 86022 Poitiers cedex, France

\begin{abstract}
Self-sustained tones are encountered when a flow passes two orifice plates, sufficiently close together, inside a duct. The upstream orifice plate creates a confined jet which impinges on the edge of the downstream orifice plate. The shear layer oscillations (coherent structures) of the confined jet in the presence of a resonant acoustic field (pipe mode), constitute the main features of cavity tones. The tones are synchronized by the pipe modes. When the flow velocity is varied for a fixed geometry, frequency jumps from one mode to another are observed. Pressure spectra showing intense acoustic energy localised in narrow bands near pipe mode frequencies are measured. We emphasize the existence of transition flow regimes during which quadratic non-linear coupling between spontaneously excited modes can take place. A digital bispectral analysis (bicoherence function) is used to quantify the degree of nonlinear interactions of acoustic waves of finite amplitude in the so-called transition region.
\end{abstract}

\section{INTRODUCTION.}

La présente étude concerne les bruits acoustiques auto-entretenus créés par un écoulement cisaillé venant frapper un obstacle. On met ici l'accent sur la présence de fortes interactions non-linéaires entre ondes acoustiques d'amplitude finie. L'outil adapté pour identifier ces interactions est la fonction de bicohérence, qui mesure la cohérence de phase entre trois composantes de Fourier. L'intérêt principal que nous voulons souligner est la mise en évidence de pics très énergétiques à des fréquences qui ne sont pas celles que l'on attend par des analyses classiques d'instabilité de couche cisaillée d'une part, par des calculs de modes acoustiques du circuit d'autre part.

L'installation expérimentale est celle d'un jet confiné d'air à basse vitesse (Mach inférieur à 0.10) provenant d'un diaphragme amont, qui traverse un diaphragme aval proche (distance: 1 à 4 fois le diamètre du diaphragme).

Dans cette configuration d'écoulement interne cisaillé rencontrant un obstacle, le mécanisme d'autoentretien du bruit acoustique prend en compte le détachement tourbillonnaire dans le jet et l'onde acoustique de retour liée à la déviation des tourbillons par l'obstacle.

Nous nous intéressons surtout aux interactions non-linéaires qui se manifestent dans des zones étroites de vitesse dites de transition, que nous définissons ainsi:

Lorsqu'on fait varier la vitesse, pour une géométrie donnée, le bruit est à peu près un son pur de fréquence fixe avec ses harmoniques dans un certain intervalle de vitesse, puis saute brusquement sur une autre fréquence pour un autre intervalle de vitesse.

Entre deux intervalles consécutifs de vitesse se situe une zone étroite appelée "saut" ou "zone de transition", où l'énergie sonore est distribuée sur de multiples pics très énergétiques qui sont le résultat d'interactions non linéaires de type quadratique entre ondes acoustiques d'amplitude finie. 


\section{INSTALLATION EXPÉRIMENTALE.}

L'installation est la suivante:
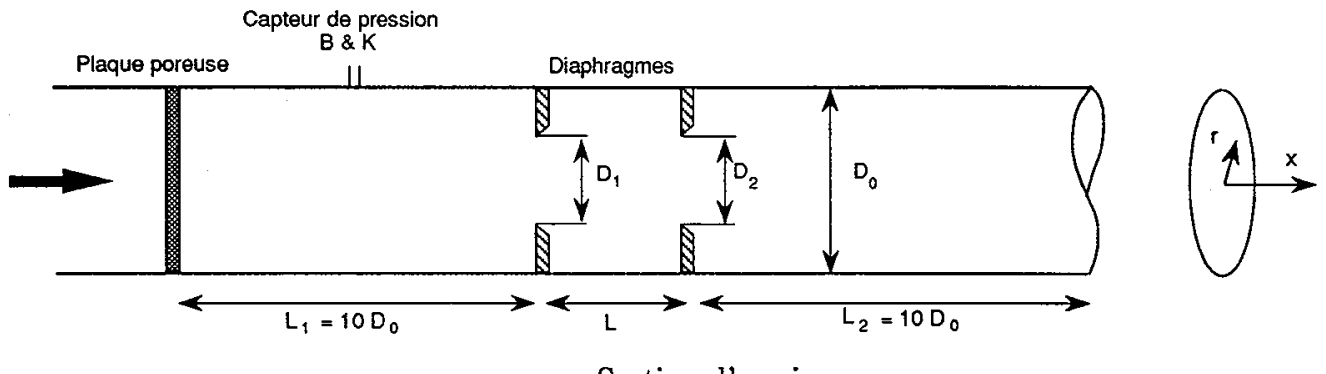

Section d'essai

L'écoulement a lieu à faible vitesse (nombre de Mach inférieur à 0.1 dans notre gamme expérimentale). Un capteur de pression à la paroi en amont permet d'enregistrer le signal acoustique. La zone expérimentale est protégée du circuit d'alimentation par un système d'amortissement non représenté ici.

\section{BRUIT AUTO-ENTRETENU.}

Les oscillations de la couche de cisaillement du jet confiné issu du $1^{e r}$ diaphragme et le champ acoustique résonnant sont en interaction par un mécanisme décrit dans la référence [2].

Lorsqu'un mode du circuit est excité, la couche de cisaillement,très sensible aux ondes acoustiques, se met à osciller à la même fréquence, régulant ainsi un détachement de tourbillons. Ceux-ci frappent le second diaphragme et transfèrent alors de l'énergie au champ acoustique qui deviınt suffisament fort pour exciter de nouveau la couche de cisaillement. On est en présence d'un bruit auto-entretenu.

\section{INTERACTIONS NON LINÉAIRES ENTRE ONDES ACOUSTIQUES D'AMPLI- TUDE FINIE. \\ OUTIL ADAPTÉ : LA FONCTION DE BICOHÉRENCE.}

\subsection{La fonction de bicohérence:}

La fonction de bicohérence $b^{2}\left(f_{1}, f_{2}\right)$ est ici l'outil adapté pour distinguer sur la densité spectrale un pic d'énergie qui est un mode naturel, d'un pic résultant d'une interaction non-linéaire entre deux modes.

Elle est définie par :

$$
b^{2}\left(f_{1}, f_{2}\right)=\frac{\left|B\left(f_{1}, f_{2}\right)\right|^{2}}{S_{x x}\left(f_{1}\right) S_{x x}\left(f_{2}\right) S_{x x}\left(f_{1}+f_{2}\right)}
$$

avec

$$
B\left(f_{1}, f_{2}\right)=E\left[X\left(f_{1}\right) X\left(f_{2}\right) X^{*}\left(f_{1}+f_{2}\right)\right]
$$

et

$$
S_{x x}(f)=E\left[X^{*}(f) X^{*}(f)\right]
$$

$\mathrm{X}(\mathrm{f})$ est la transformée de Fourier du signal (ici, la fluctuation de pression donnée par le capteur), E est l'espérance mathématique.

$b^{2}\left(f_{1}, f_{2}\right) \approx 1$ ou 0 signifie respectivement qu'il y a, ou non, interaction non linéaire entre les ondes $f_{1}$ et $f_{2}$. 


\subsection{Les interactions non linéaires.}

4.2.1 Un "cas école":

Voici un exemple de densité spectrale du champ de pression acoustique où figurent trois pics énergétiques dont un est le résultat d'une interaction non linéaire, comme le montre la fonction de bicohérence associée
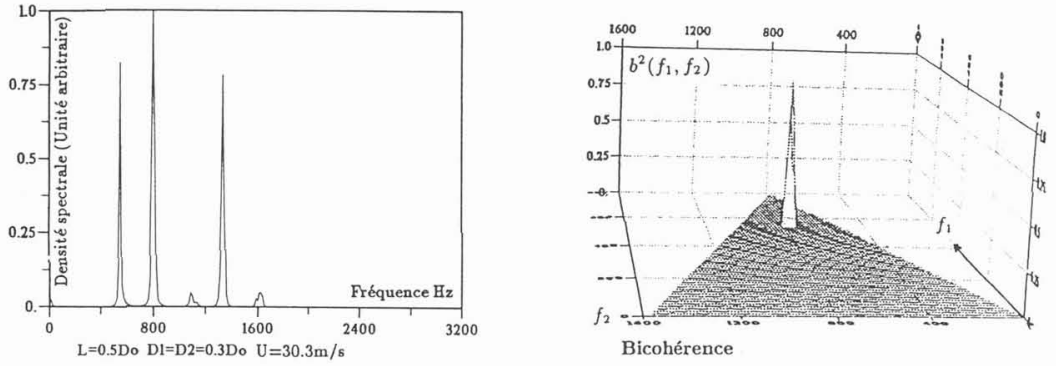

Spectre de pression

4.2.2 Un cas complexe :

Une multitude d'interactions non linéaires identifiées par les nombreux pics de la fonction de bicohérence.
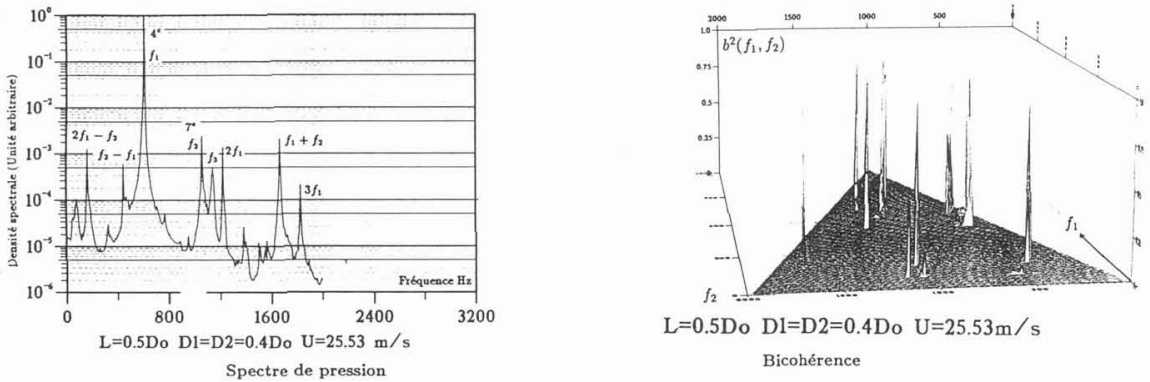

4.2.3 L'évolution type du phénomène lorsqu'on passe d'un mode à un autre :

Etude par l'analyse spectrale et bispectrale de la "zone de transition". Entre une gamme de vitesses et la suivante (pour lesquelles le bruit est pour chacune des deux gammes un son "pur" avec ses harmoniques) se situe une étroite zone de vitesses où la densité spectrale présente une multitude de pics résultant de multiples interactions non linéaires.

L'évolution type de la densité spectrale, ainsi que de la fonction de bicohérence, est donnée dans l'exemple suivant
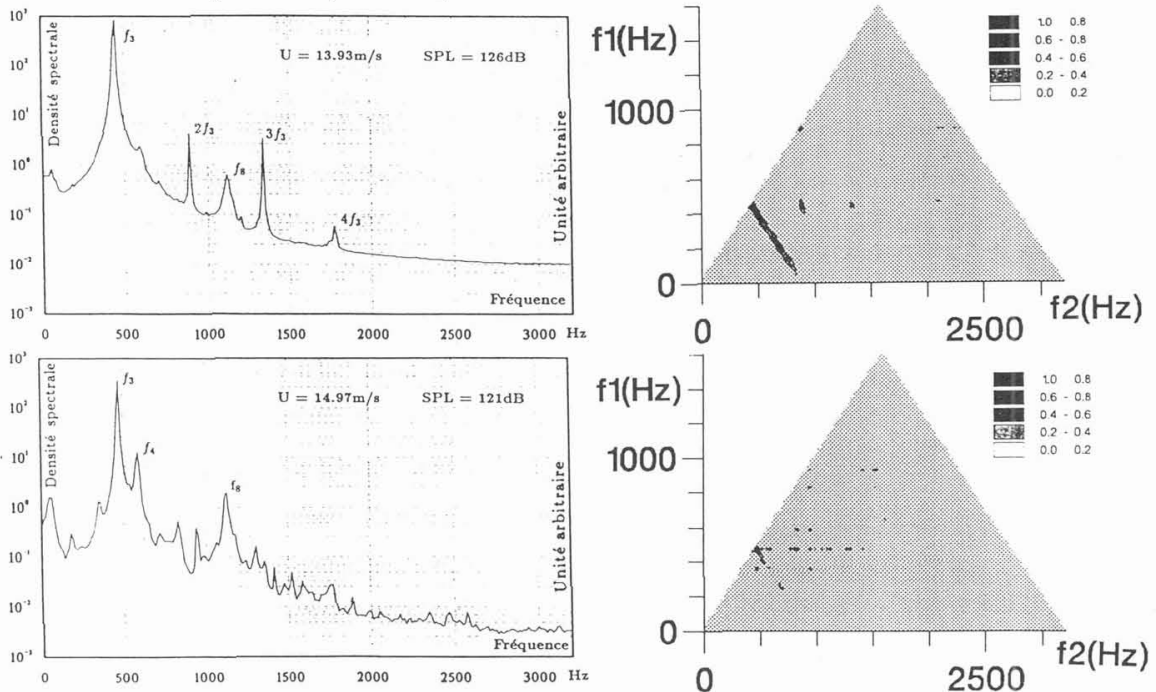

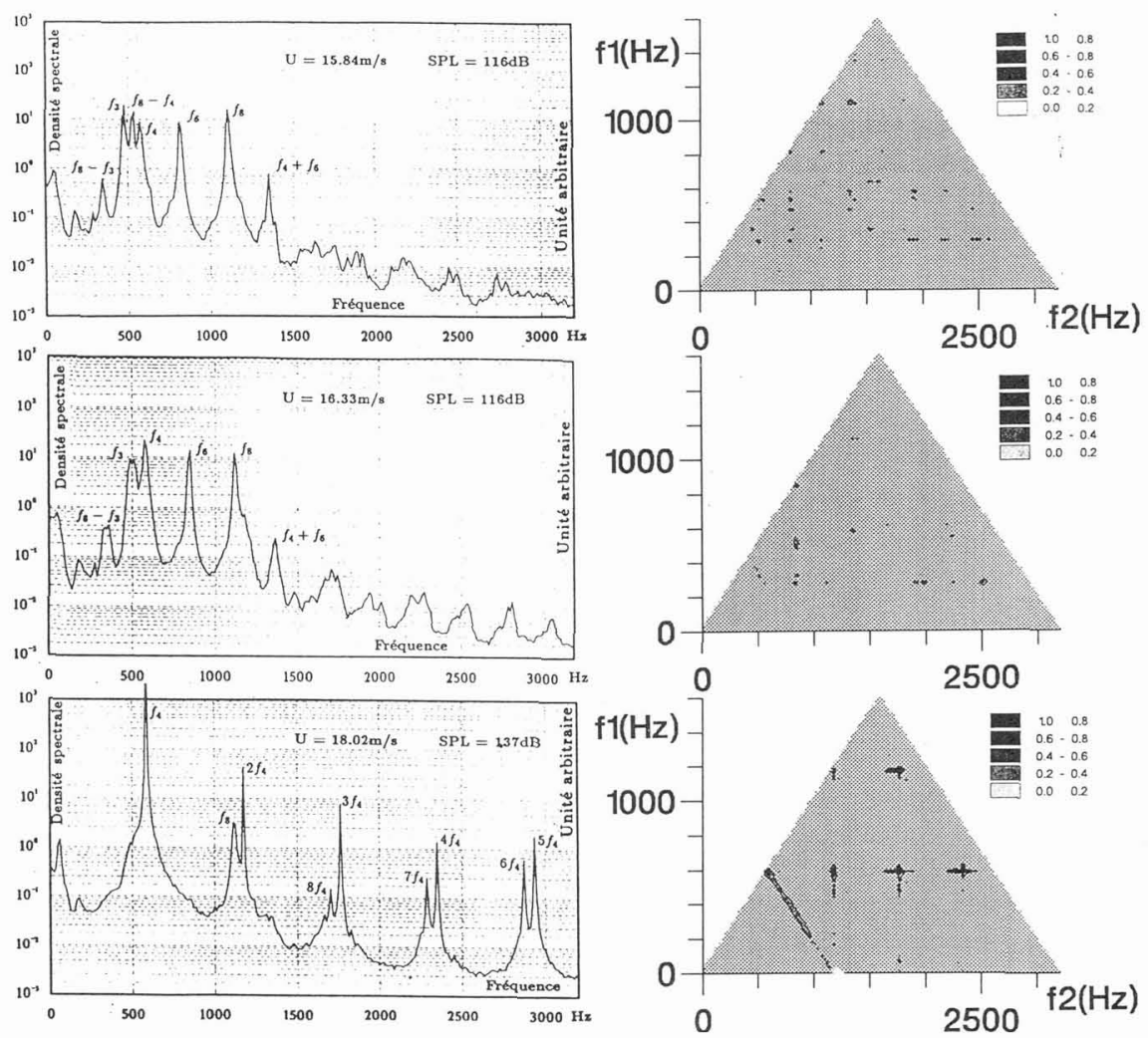

CONCLUSION:

Les interactions non linéaires entre ondes acoustiques, sont clairement mises en évidence à l'aide d'un outil de traitement du signal adapté : la fonction de bicohérence qui, pour l'essentiel, mesure la cohérence de phase entre trois composantes de Fourier.

Nous avons observé que ces interactions sont présentes, surtout lors du bref intervalle "de transition" entre un mode acoustique et un autre mode, lorsqu'on fait varier la vitesse de l'écoulement. C'est à dire encore que pour un mode bien établi, les interactions non linéaires conduisent seulement aux harmoniques de ce mode, alors que dans un "intervalle de transition", les interactions non linéaires se font entre deux ou plusieurs modes propres du circuit.

Les interactions non linéaires sont notables, lorsque le niveau du bruit est important, ce qui est normal puisqu'il s'agit d'interactions non linéaires entre ondes acoustiques d'amplitude finie. Elles sont également notables, dans le cas de notre configuration géométrique, lorsque les deux diaphragmes sont très rapprochés.

L'analyse des densités spectrales du bruit sonore, jointe à une analyse fine et détaillée des fonctions de bicohérence, permet de mettre en évidence la multitude des interactions non linéaires. Dans un intervalle dit "de transition", l'allure complexe et désordonnée des densités spectrales est due au grand nombre d'interactions non linéaires. L'énergie sonore est alors distribuée sur de très nombreuses fréquences, dont certaines, qui peuvent être très énergétiques, sont "inattendues" si l'on n'a pas tenu compte des éventuelles interactions non linéaires.

Les applications sont nombreuses: aéronautique, moyens de transport, combustion,...

\section{REFERENCES}

[1] I.S. Soreefan 1993 Contribution à l'étude des bruits auto-entretenus créés par un jet confiné rencontrant un obstacle. Thèse Université de POITIERS.

[2] I.S. Soreefan, R. Henry \& C. Favé 1993 Self-sustained low frequency tones generated by a confined jet impinging on an obstacle at low Mach number. J. Low Frequency Noise and Vibration 12(2), 30-35.

(3) K. Hourigan, M.C. Welsh, M.C. Thompson \& A.N. Stokes 1990. J. Fluids and Structures 4, 345-370.

[4] J.C. Bruggeman, A. Hirschberg, M.E.H. van Dongen, A.P.J. Wijnands \& J. Gorter 1991. J. Sound and Vibration 150(3), 371-393.

[5] Y.C Kim \& E.J. Powers 1979. IEEE Transactions On Plasma Science PS-7(2), 120-131. 